\title{
Direct and indirect effects of insulin on hepatic glucose production in diabetic depancreatized dogs during euglycemia
}

\author{
Neehar Gupta ${ }^{1, *}$, Edward Park ${ }^{1}$ *, Harmanjit Sandhu ${ }^{1}$, Tracy Goh ${ }^{1}$, Vaja Tchipashvili ${ }^{1}$ and Adria Giacca ${ }^{1,2}$ \\ Departments of ${ }^{1}$ Physiology and ${ }^{2}$ Medicine, Medical Sciences Building, University of Toronto, Toronto, Ontario M5S 1A8, Canada \\ (Requests for offprints should be addressed to A Giacca; Email: adria.giacca@utoronto.ca) \\ *(N Gupta and E Park equally contributed to this work)
}

\begin{abstract}
Insulin suppresses glucose production (GP) via both extrahepatic (indirect) and hepatic (direct) effects. We have shown that the direct effect, undetectable in moderately hyperglycemic diabetic dogs, is restored by insulin-induced euglycemia. The first aim of the present study was to determine whether euglycemia per se, and not the excess insulin needed to obtain it, restores the direct effect of insulin on GP. Basal insulin was given portally in depancreatized dogs to attain only moderate hyperglycemia, then an additional insulin was given portally or peripherally to match the peripheral insulin levels and thus to obtain a greater hepatic insulinization with portal delivery. Plasma glucose was allowed to fall to euglycemia before a euglycemic clamp was performed. During euglycemia, there was a tendency $(P=0 \cdot 075)$ for greater suppression of GP by portal than peripheral insulin. Also, there was a significantly different
\end{abstract}

effect of time $(P=0 \cdot 01)$ on GP in the two groups, with greater suppression over time in the portal group. The second aim was to test the hypothesis that because of inadequate hepatic insulinization and consequent lack of direct inhibition of GP, peripheral insulin replacement requires peripheral hyperinsulinemia to achieve euglycemia. Portal or peripheral insulin was given to achieve euglycemia and basal GP, and insulin levels were measured. More peripheral insulinemia was required with peripheral than portal insulin replacement to maintain similar euglycemia and GP. Our conclusions are as follows: (1) euglycemia per se is sufficient to acutely restore the direct effect of insulin on GP and (2) at euglycemia, peripheral replacement of insulin, as in insulin-treated diabetes, results in peripheral hyperinsulinemia but unchanged basal GP.

Journal of Endocrinology (2006) 190, 695-702

\section{Introduction}

It is well established that insulin has direct and indirect inhibitory effects on glucose production (GP). The direct effect is due to interaction of hepatic sinusoidal insulin with the hepatocyte insulin receptor (Lewis et al. 1996, Sindelar et al. 1996, 1997, 1998, McCall et al. 1998, Staehr et al. 2001) and the indirect effect is due to insulin's actions on extrahepatic tissues, such as muscle and adipose tissue (Prager et al. 1987, Ader \& Bergman 1990, Lewis et al. 1996, Sindelar et al. 1996, 1997, 1998, McCall et al. 1998, Staehr et al. 2001), $\alpha$ cells (Giacca et al. 1997), and brain (Obici et al. 2002).

The importance of the direct and indirect effects of insulin in the regulation of GP appears to be different in the non-diabetic versus diabetic individuals. Our laboratory has previously reported a lack of the direct effect of insulin in suppressing GP in moderately hyperglycemic depancreatized dogs as suppression of GP was proportional to peripheral, but not hepatic sinusoidal insulin levels (Giacca et al. 1992, 1999). Furthermore, in our collaborative studies, the direct effect of insulin on steadystate GP was undetectable in humans with type 2 diabetes and moderate hyperglycemia (Lewis et al. 1999).
In a subsequent study (Gupta et al. 2002), portal insulin infusion (POR) matching the peripheral insulin levels, obtained with half dose peripheral infusion (1/2 PER), led to the greater suppression of GP than 1/2 PER under conditions of euglycemia, consistent with the greater hepatic insulinization obtained with portal insulin delivery. This finding demonstrated that euglycemia can restore the direct suppressive effect of insulin on GP, indicating that hyperglycemia may impair this effect. Hyperglycemia can inhibit glycogenolysis, thereby shifting GP to gluconeogenesis (Rossetti et al. 1993). This can, in turn, diminish the direct suppressive effect of insulin on GP, which mainly consists of glycogenolysis (Sindelar et al. 1996). In the study by Gupta et al. (2002), additional insulin was infused to lower blood glucose from hyperglycemia to euglycemia during the basal period; therefore, it still remains to be demonstrated that euglycemia per se, rather than the extra amount of insulin infused, induced the direct effect of insulin observed. Furthermore, it is not clear whether at euglycemia, the direct effect of insulin has an insulin-sparing effect, i.e. less peripheral insulin is required to maintain euglycemia with portal replacement (POR REPL) compared with peripheral 
insulin replacement (PER REPL). The latter is of clinical importance as hyperinsulinemia is a suspected risk factor for atherosclerosis and some types of cancer (Despres et al. 1996, Giovannucci 2001). The objective of the present study was to address these two issues.

More specifically, we wished to determine whether portal infusion of insulin suppresses GP to a greater extent than peripheral insulin infusion, when euglycemia is reached after a basal period of moderate hyperglycemia. Therefore, we reproduced the experimental conditions of Giacca's study (Giacca et al. 1992; moderate hyperglycemia obtained by subbasal insulin replacement followed by additional infusion of insulin at the same rates as in Giacca's study); however, instead of clamping plasma glucose at the basal hyperglycemic levels, we allowed the plasma glucose levels to decline during the same-rate insulin infusion in order to obtain a selective difference in plasma glucose between the two studies (as well as between the first and the last part of the experiment in the present study). As in Giacca's study (Giacca et al. 1992), POR was compared with peripheral infusion at approximately half the rate (1/2 PER), as first pass hepatic extraction of portally delivered insulin is approximately $50 \%$. We also wished to determine whether the POR REPL dose of insulin required to maintain euglycemia leads to lower peripheral insulin levels, compared with the PER-REPL dose of insulin. We hypothesized that (1) POR suppresses GP to a greater extent than 1/2 PER when euglycemia is reached and (2) lower peripheral circulating insulin is needed with POR-REPL than with PER-REPL to attain euglycemia.

\section{Materials and Methods}

\section{Experimental animals and preparation}

The studies were performed on six post-absorptive depancreatized male dogs (model of type 1 diabetes mellitus) weighing $25-35 \mathrm{~kg}$. Total pancreatectomy followed by cannulation of portal vein, jugular vein, and carotid artery was performed under general anesthesia as previously described (Gupta et al. 2002). The animal model and preparation were the same as in our previous studies (Giacca et al. 1992, 1999, Gupta et al. 2002). All procedures were in accordance with the Canadian Council of Animal Care Standards and were approved by the Animal Care Committee of the University of Toronto.

The dogs received a mixture of dry chow mixed with canned meat once a day. The food was supplemented with folic acid and iron. In addition, pancreatic enzymes (Cotazym, Organon Canada, Toronto, ON, Canada) were given orally to replace the lost exocrine function of the pancreas. Regular porcine (Iletin II regular insulin, Eli Lilly Co.) and NPH porcine insulin in an approximate ratio of 1:2 were injected subcutaneously. Glycemia was measured using a glucometer (Bayer Inc.). The insulin dose was adjusted according to blood glucose, in order to maintain fasting glycemia at $<11 \mathrm{mmol} / \mathrm{l}$. Body weight, body temperature, hematocrit, stools, and food intake were monitored regularly.

Only dogs that were healthy, with a hematocrit above 35\% and under relatively well-controlled diabetes (glucose $<11 \mathrm{mmol} / \mathrm{l})$, were allowed to undergo experiments. The dogs received a normal amount of food, the day before the experiment. The regular insulin dose was unaffected and the NPH insulin was reduced to half or one-third of the previous day's dose. The experiments were performed after an overnight fast of at least $18 \mathrm{~h}$. Of the six dogs, five underwent Protocol 1 and six underwent Protocol 2 (all paired experiments). This discrepancy in the number of dogs used for each protocol was due to occlusion of the portal catheter in one of the dogs, which had been used for Protocol 2 first. The experiments were performed in random order.

\section{Experimental protocol (Fig. 1)}

Protocol 1 Similar to our previous studies, at the onset of the experiment, the dogs were hyperglycemic $(>20 \mathrm{mmol} / \mathrm{l})$ and a high POR rate was administered, which was gradually reduced to a basal rate. Instead of obtaining euglycemia, as in Gupta's study (Gupta et al. 2002), the goal was to reach moderate hyperglycemia (9-11 $\mathrm{mmol} / \mathrm{l})$ as in earlier studies (Giacca et al. 1992). The portal dose to achieve moderate hyperglycemia during the basal period (Fig. 1) was $1 \cdot 8 \pm 0 \cdot 2$ (POR) and $1 \cdot 4 \pm 0 \cdot 2 \mathrm{pmol} / \mathrm{kg}$ per min $(1 / 2 \mathrm{PER} ; \mathrm{NS})$. When glucose levels decreased below $16 \mathrm{mmol} / \mathrm{l}(\sim 80 \mathrm{~min}$ after the insulin infusion was started), a bolus of tracer $\left(7 \cdot 77 \times 10^{7}\right.$ d.p.m. of $3-\left[{ }^{3} \mathrm{H}\right]$ glucose; New England Nuclear, Boston, MA, USA) followed by a continuous tracer infusion $(5 \cdot 55 \times$ $10^{5}$ d.p.m./min) was given to enable the measurements of GP and glucose utilization (GU). Approximately $120 \mathrm{~min}$ were required to reach moderate hyperglycemia after the tracer infusion was initiated, and an additional $60 \mathrm{~min}$ period of constant moderate hyperglycemia elapsed before the first basal sample was taken at $-40 \mathrm{~min}$. At that time, the insulin infusion (portal insulin replacement) had been kept fixed at a basal rate for $\geq 30 \mathrm{~min}$. Both the portal insulin replacement and the tracer infusion were continued throughout the experiment. Basal samples were taken every $10 \mathrm{~min}$ for $40 \mathrm{~min}$. After 40-min basal period, an additional insulin dose $(5.4 \mathrm{pmol} / \mathrm{kg}$ per min; as in Giacca's study (Giacca et al. 1992)) was administered either portally or peripherally at half the portal dose to match the peripheral insulin levels obtained with the POR treatment (due to $\sim 50 \%$ insulin extraction by the liver) and also to obtain a large difference in the estimated hepatic insulin levels. The dose that matched the peripheral insulin levels was slightly lower than $50 \%(2 \cdot 34 \mathrm{pmol} / \mathrm{kg}$ per $\mathrm{min})$ of the portal dose and was determined experimentally (Gupta et al. 2002). Unlike Giacca's study (Giacca et al. 1992), in which plasma glucose was clamped at the basal hyperglycemic level, in this study, the glucose levels were allowed to fall until euglycemia was achieved and then plasma glucose was clamped for the remainder of $3 \mathrm{~h}$ at euglycemia with a variable exogenous glucose infusion (50\% dextrose, Abbott Laboratories), adjusted according to 


\section{Experimental Protocol}

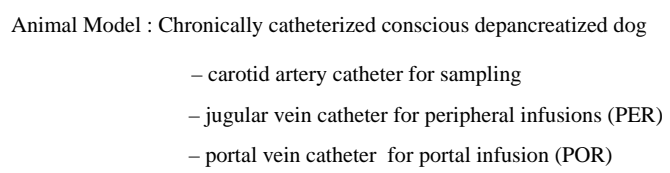

Protocol 1

\begin{tabular}{|c|c|}
\hline $\begin{array}{c}\text { Portal insulin to achieve } \\
\text { moderate hyperglycemia }\end{array}$ & Continuation of portal insulin replacement (POR REPL) \\
\hline $3-\left[{ }^{3} \mathrm{H}\right]$ glucose & Continuation of $3-\left[{ }^{3} \mathrm{H}\right]$ glucose infusion \\
\hline & Insulin: $5.4 \mathrm{pmol} / \mathrm{kg} . \mathrm{min}(\mathrm{POR})$ or \\
$2.34 \mathrm{pmol} / \mathrm{kg} \cdot \mathrm{min}(\sim 1 / 2 \mathrm{PER})$
\end{tabular}

\section{Protocol 2}

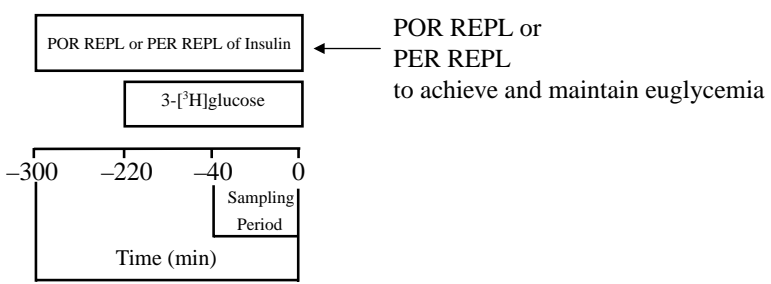

Figure 1 Experimental design. Briefly, in Protocol 1, depancreatized dogs were given a sub-basal insulin infusion to obtain moderate hyperglycemia in the basal state. Thereafter, additional insulin was infused portally or peripherally to match the peripheral insulin levels and the glucose levels were allowed to fall to euglycemia. Protocol 2 involves short experiments with portal or peripheral basal insulin replacement to achieve basal euglycemia (note: the time indicated is not to scale).

plasma glucose concentrations determined every $5 \mathrm{~min}$. Furthermore, whereas in the Giacca's study (Giacca et al. 1992), a 'hot glucose' infusion was required to maintain the plasma glucose specific activity; in this study, since GU changed to a lesser extent than GP, 'cold glucose' was needed to maintain plasma glucose specific activity constant. Blood samples were taken at repeated intervals for the same determinations of plasma radioactivity, metabolites, and hormones (Fig. 1, top panel).

Protocol 2 A second study was conducted in which insulin was infused portally or peripherally from the onset of the experiment and then gradually reduced as described for protocol 1. However, the purpose was to achieve euglycemia, as in Gupta's study (Gupta et al. 2002). The insulin infusion rates required to achieve euglycemia (Fig. 1) were $4 \cdot 1 \pm 0 \cdot 5$ (POR REPL) and $2 \cdot 0 \pm 0 \cdot 2 \mathrm{pmol} / \mathrm{kg}$ per min (PER REPL). As described for protocol 1, a primed tracer infusion was initiated when glucose levels were $<16 \mathrm{mmol} / 1$ to enable the measurement of glucose turnover. We reached euglycemia approximately $120 \mathrm{~min}$ thereafter and an additional $60 \mathrm{~min}$ elapsed before the first basal sample was taken at $-40 \mathrm{~min}$. At that time, the insulin infusion had been kept fixed at a basal rate for $30 \mathrm{~min}$. We measured basal GP and GU, metabolites and hormones up to time 0 . At time 0 , the experiment was interrupted (we did not perform the additional POR or PER; Fig. 1, bottom panel).

\section{Laboratory methods}

Plasma glucose concentrations were measured by the glucose oxidase method (Kadish \& Sternberg 1969) on a glucose analyzer (Glucose Analyzer II, Beckman Instruments, Fullerton, CA, USA). The RIA for insulin and glucagon was performed using kits from Pharmacia and Diagnostic Products respectively. The coefficients of variation of the assays were $<7$ and $<16 \%$ respectively. The free fatty acid (FFA) concentrations were determined with the fluorometric method of Miles et al. (1983). For the determination of $3-\left[{ }^{3} \mathrm{H}\right]$-glucose radioactivity, plasma was deproteinized in equal volumes of $5 \%(\mathrm{w} / \mathrm{v})$ zinc sulfate and $0 \cdot 15 \mathrm{M}$ barium 
hydroxide (Sigma Diagnostics). An aliquot of the supernatant was evaporated to eliminate tritiated water. After addition of $1 \mathrm{ml}$ double distilled water, $10 \mathrm{ml}$ liquid scintillation solution (Ready Safe, Beckman) was added, the vials were vortexed, and the radioactivity from $3-\left[{ }^{3} \mathrm{H}\right]$-glucose was measured in a $\beta$ scintillation counter (Camberra Packard, Meriden, CT, USA). Aliquots of the infused glucose tracer and of the labeled glucose infusate were diluted with non-radioactive plasma of the same dog and assayed together with the plasma samples.

\section{Calculations}

GP was calculated as the endogenous rate of appearance measured with 3-[ $\left.{ }^{3} \mathrm{H}\right]$-glucose using Steele's methods (Finegood et al. 1987). GU was the tracer-determined rate of disappearance ( $\mathrm{Rd}$ ), whereas glucose metabolic clearance rate (MCR) was calculated as $\mathrm{Rd} /$ [plasma glucose] (Radziuk \& Lickley 1985). Rd corresponded to GU, and plasma clearance rate of glucose to glucose MCR, because plasma glucose levels were below the renal threshold for glucose in dogs (Bjorkman et al. 1988). Data were smoothed with the optimal segments routine using the optimal error algorithm (Bradley et al. 1993).

In Protocol 1, we calculated the first-pass hepatic insulin extraction (HIE) of portally delivered insulin using the following formula:

$\operatorname{HIE}(\%)=\frac{\Delta \mathrm{INF}_{\mathrm{POR}}-\left(\Delta[\mathrm{Ins}]_{\mathrm{PE}-\mathrm{POR}} \times \mathrm{INF}_{1 / 2 \mathrm{PER}} / \Delta[\mathrm{Ins}]_{\mathrm{PE}-1 / 2 \mathrm{PER}}\right)}{\Delta \mathrm{INF}_{\mathrm{POR}}}$ $\times 100$

where; $\Delta \mathrm{INF}_{\mathrm{POR}}$ is the additional portal infusion during the POR treatment, $\Delta[\text { Ins }]_{\text {PE-POR }}$ is the increment in the peripheral insulin level due to $\Delta \mathrm{INF}_{\mathrm{POR}}, \mathrm{INF}_{1 / 2} \mathrm{PER}$ is the $1 / 2$ PER in the paired 1/2 PER treatment in the same dog, and $\Delta[\text { Ins }]_{\mathrm{PE}-1 / 2 \mathrm{PER}}$ is the increment in the peripheral insulin level due to $\mathrm{INF}_{1 / 2}$ PER. $\mathrm{INF}_{1 / 2}$ PER $/ \Delta[\operatorname{Ins}]_{\mathrm{PE}-1 / 2}$ PER represents plasma insulin clearance rate (PCR) and PCR $X$ $\Delta[\text { Ins }]_{\text {PE-POR }}$ is the systemic appearance of portally delivered insulin. In Protocol 2, HIE\% was calculated using the same formula, except $I_{N F_{P O R-R E P L}}$ replaced $\Delta \mathrm{INF}_{\mathrm{POR}}$ and $I_{\text {INFR-REPL }}$ replaced $I_{\text {INF }} / 2$ PER.

\section{Statistical analysis}

The data are expressed as mean \pm s.E.M. Two-way ANOVA or paired $t$-test was carried out for differences between experimental groups as appropriate. Data were also analyzed within each group for differences between the experimental periods. Calculations were performed with SAS software (SAS Statistical Analysis System, Cary, NC, USA).

\section{Results}

\section{Protocol 1}

The following results are based on an $n=5$ for POR and $1 / 2$ PER. The basal peripheral insulin levels were $40 \pm 5$ and $33 \pm$ $1 \mathrm{pmol} / \mathrm{l}$ (not significant; NS) in the POR and 1/2 PER groups respectively (Fig. 2A). The additional POR in the POR treatment raised the peripheral insulin levels to $\sim 140 \mathrm{pmol} / 1$, which was not different from peripheral insulin levels observed in 1/2 PER (Fig. 2A). Insulin extraction by the liver was calculated to be $55 \%$.

The basal plasma glucose levels were maintained constant at moderate hyperglycemia $(\sim 10 \mathrm{mmol} / \mathrm{l})$. Throughout the course of the experiment, the glucose levels declined equally, until euglycemia was reached between 90 and $105 \mathrm{~min}$. Thereafter, the glucose levels were clamped for the remainder of the experiment (Fig. 2B). Plasma glucose specific activity increased slightly during the insulin infusion (this is why 'hot
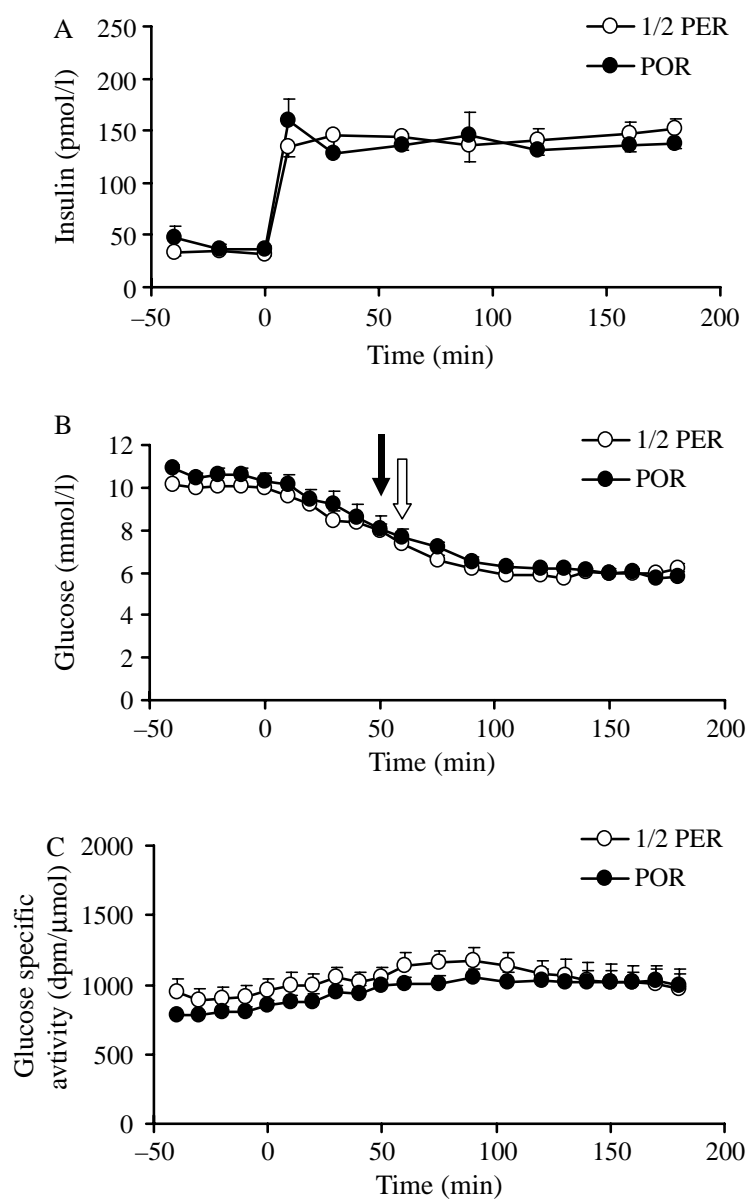

Figure 2 (A) Plasma insulin, (B) glucose, and (C) glucose-specific activity during Protocol 1 (as described in Fig. 1). Open and closed arrows indicate the start of glucose infusion in the $1 / 2$ peripheral insulin infusion (PER) and portal insulin infusion (POR) groups respectively. Results are mean \pm S.E.M. 
glucose' infusion was not needed). The increase was $\sim 20$ and $13 \%$ in the POR and $1 / 2$ PER groups respectively (Fig. 2C).

The glucose infusion rates necessary to maintain euglycemia were slightly, but not significantly, higher in $1 / 2$ PER than POR $(P=0 \cdot 088$; Fig. 3A). At $180 \mathrm{~min}$, glucose infusion rate reached $15 \cdot 4 \pm 4 \cdot 3 \mu \mathrm{mol} / \mathrm{kg}$ per min in $1 / 2 \mathrm{PER}$ compared with $11 \cdot 6 \pm 2 \cdot 7 \mu \mathrm{mol} / \mathrm{kg}$ per min in POR.

The GU in the basal periods was similar in the POR and 1/2 PER treatments respectively. Because of the glucose decline, GU increased only marginally with both POR and $1 / 2$ PER except in the last $40 \mathrm{~min}$, when GU was significantly higher in $1 / 2$ PER $(P<0 \cdot 001$; Fig. 3B). MCR rose with both POR and $1 / 2$ PER (Fig. 3C). In the last $40 \mathrm{~min}, \mathrm{MCR}$ was greater in $1 / 2 \mathrm{PER}$ than $\mathrm{POR}(P<$ $0 \cdot 01)$. At $180 \mathrm{~min}, \mathrm{GU}$ was $26 \cdot 9 \pm 4 \cdot 2 \mu \mathrm{mol} / \mathrm{kg}$ per min in $1 / 2 \mathrm{PER}$ compared with $22 \cdot 3 \pm 2 \cdot 2 \mu \mathrm{mol} / \mathrm{kg}$ per $\mathrm{min}$ in POR, whereas MCR was $4 \cdot 44 \pm 0 \cdot 78$ and $3 \cdot 89 \pm 0 \cdot 47 \mathrm{ml} /$ $\mathrm{kg}$ per min respectively.

Basal endogenous GP was $18 \cdot 1 \pm 1 \cdot 5$ and $18 \cdot 2 \pm$ $1 \cdot 7 \mu \mathrm{mol} / \mathrm{kg}$ per min in the POR and $1 / 2 \mathrm{PER}$ treatments respectively. When the additional insulin was given, GP was suppressed to the same extent for most of the experiment. However, during the last $20 \mathrm{~min}$, the POR treatment tended to suppress GP to a greater extent than the 1/2 PER treatment $(P=0 \cdot 075$; Fig. 3D). At $180 \mathrm{~min}$, GP was $13 \cdot 5 \pm$
$2.5 \mu \mathrm{mol} / \mathrm{kg}$ per min in $1 / 2$ PER compared with $9 \cdot 36 \pm$ $1.5 \mu \mathrm{mol} / \mathrm{kg}$ per $\mathrm{min}$ in POR, which was also not significantly different between treatments. However, when only the portal treatment was considered, the decrease in GP from 90 or $105 \mathrm{~min}$ to the $180 \mathrm{~min}$ time-point was very close to statistical significance $(P=0 \cdot 055$ or $0 \cdot 057$ respectively). In addition, after $90 \mathrm{~min}$ there was a significant interaction between time and treatment $(P=0 \cdot 01)$, which is evidence for a significantly different effect of time on GP with POR versus PER (i.e. separation of the two groups over time).

The basal FFA levels were not significantly different between the POR and 1/2 PER groups. During the additional insulin infusion, the FFA levels declined to a slightly greater extent in $1 / 2 \operatorname{PER}(P<0 \cdot 01$; Table 1$)$. The basal glucagon levels were similar in the POR and 1/2 PER groups. During the additional insulin infusion, the glucagon levels declined modestly $(P<0 \cdot 05)$ to a slightly lower level in $1 / 2$ PER than POR $(P<0 \cdot 01$; Table 1$)$.

\section{Protocol 2}

The following results are based on $n=6$ for POR-REPL and PER-REPL. The plasma glucose levels were equal and in the euglycemic range (POR-REPL $6 \cdot 1 \pm 0 \cdot 2$, PER-REPL $6 \cdot 4 \pm 0 \cdot 1 \mathrm{mmol} / 1$, NS; Fig. 4A).
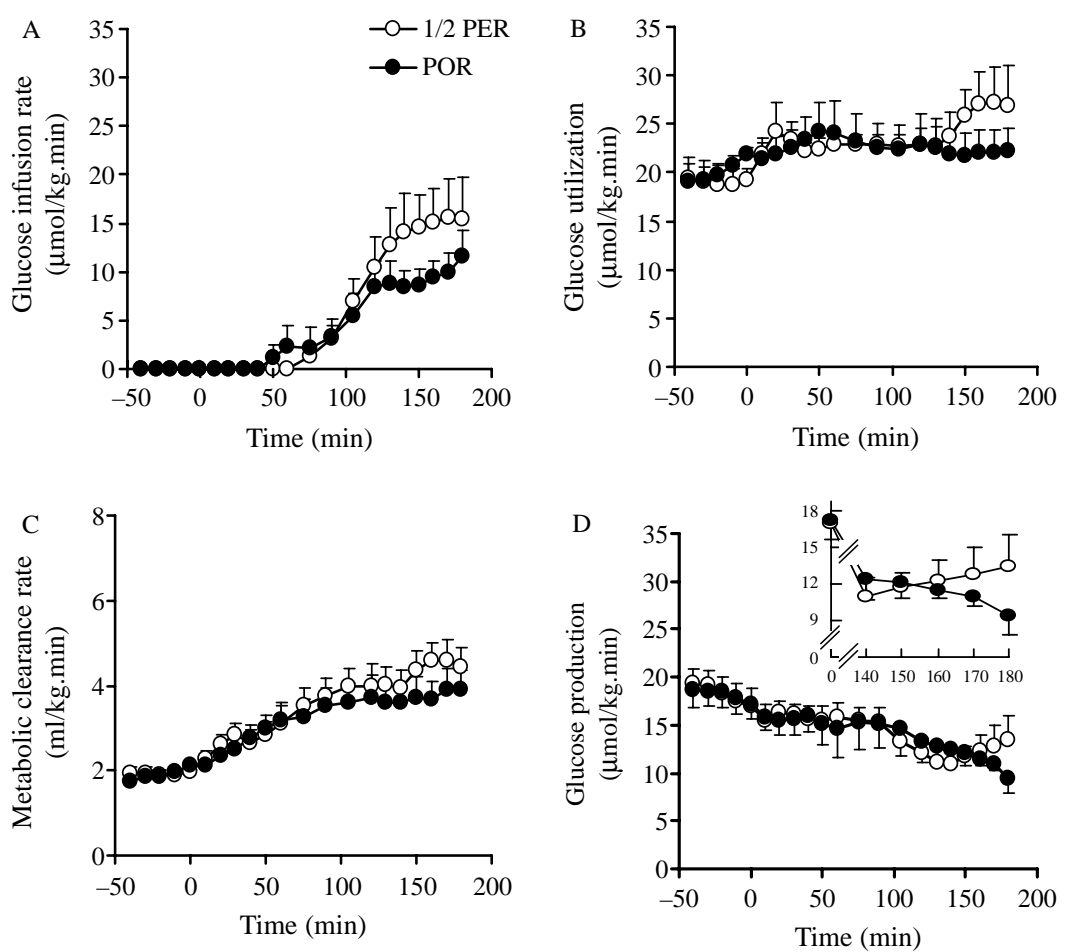

Figure 3 (A) Glucose infusion rate, (B) glucose utilization, (C) metabolic clearance rate of glucose, and (D) glucose production (GP) during Protocol 1. Protocol 1 is described in Fig. 1. The insert in Graph D depicts GP during the last 40 min of the experiment. Results are presented as mean \pm S.E.M. 
Table 1 Free fatty acid (FFA) and glucagon levels during Protocol 1

\section{1/2 PER}

FFA ( $\mu$ equiv./l)
Basal
Insulin infusion
Glucagon $(\mathrm{pg} / \mathrm{ml})$
Basal
Insulin infusion
POR

$981 \pm 86$ $524 \pm 63$

$1063 \pm 72$

$439 \pm 57^{*}$

$59 \pm 8$

$62 \pm 6$

$49 \pm 6^{*}$
1/2PER, half dose peripheral infusion; POR, portal insulin infusion. ${ }^{*} P<0 \cdot 01$ versus POR.

Endogenous GP $(\mathrm{GP}=\mathrm{GU}$ in basal steady-state conditions like those of this protocol) was not significantly different in the POR-REPL and PER-REPL treatments respectively (Fig. 4B).

The peripheral insulin levels were significantly higher in the PER-REPL $(P<0 \cdot 01)$ than in POR-REPL (PERREPL: $102 \pm 13 \mathrm{pmol} / 1$, POR-REPL $57 \pm 4 \mathrm{pmol} / 1$, Fig. 4C). Insulin extraction by the liver was $58 \cdot 3 \%$. The FFA levels tended to be lower in PER-REPL (795 \pm $94 \mu$ equiv. $/ 1)$ than POR-REPL (936 $\pm 110 \mu$ equiv./1); however, the difference was not significant $(P=0 \cdot 12$; Fig. 4D). The glucagon levels were similar in both treatments (POR-REPL 56 4 pg/ml, PER-REPL $58 \pm 4 \mathrm{pg} / \mathrm{ml}, \mathrm{NS}$ ).
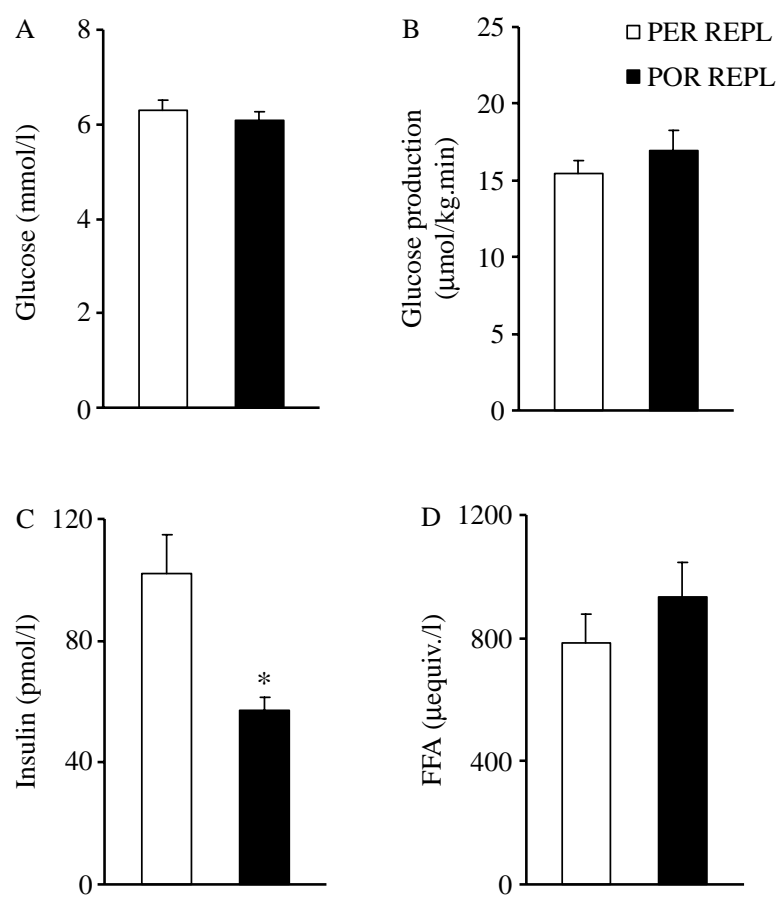

Figure 4 (A) Plasma glucose, (B) glucose production, (C) plasma insulin, and (D) plasma FFA during Protocol 2. Protocol 2 is described in Fig. 1. Results are presented as mean \pm s.E.M. ${ }^{*} P<0 \cdot 01$.

\section{Discussion}

\section{Protocol 1}

We have previously found a direct effect of insulin, i.e. greater GP suppression by POR than 1/2 PER in euglycemic (Gupta et al. 2002), but not moderately hyperglycemic dogs (Giacca et al. 1997, 1999). However, in euglycemic dogs, more insulin had to be infused in the basal state to achieve euglycemia rather than moderate hyperglycemia and this basal infusion was continued throughout the experiment (Gupta et al. 2002). Therefore, during the clamp period, not only glucose but also insulin infusion was different. It is possible that this extra insulin and not euglycemia per se could induce a direct effect. Therefore, in Protocol 1 of the present study, insulin was administered until we reached moderate hyperglycemia in the basal period, as performed previously (Giacca et al. 1992). To examine the effect of euglycemia per se on the direct effect of insulin, the glucose level was allowed to fall until euglycemia was reached. At this point, insulin levels were the same as in Giacca's study (Giacca et al. 1992) and the two studies were different only in terms of the glucose levels. Similar to Giacca's study (Giacca et al. 1992), at hyperglycemia there was no difference between treatments as GP was suppressed equally. However, near the end of the experiment, when euglycemia was reached, there was a trend towards greater suppression of GP with POR compared with 1/2 PER and a significant time-related separation of the two groups. This is contrary to Giacca's study (Giacca et al. 1992), but similar to Gupta's study (Gupta et al. 2002).

Unlike Giacca's (Giacca et al. 1992) and Gupta's (Gupta et al. 2002) studies, glucose infusion rate and GU were not the same in both groups despite the peripheral insulin levels being matched. Also, FFA and glucagon levels were slightly but significantly lower in 1/2 PER. These differences were small and their explanation is unclear. It is possible that there were random differences in peripheral insulin sensitivity between the paired experiments in the same animals (although ours was not a 'gold standard' protocol to assess peripheral insulin sensitivity because glucose levels declined, peripheral insulin and glucose levels were matched in POR and 1/2 PER groups, so differences in $\mathrm{Rd}$ and MCR should reflect differences in peripheral insulin sensitivity). This may be supported by the fact that the insulin infusion rates that were necessary to achieve euglycemia in the basal period, although not significantly different, were lower in the 1/2 PER group.

Until $150 \mathrm{~min}$, GP was suppressed to the same extent in both treatments. However, after $150 \mathrm{~min}$, the suppression of GP tended to be greater with POR than 1/2 PER despite higher FFA and glucagon levels with the POR treatment. This would indicate that a direct effect of insulin was likely to have been underestimated in the POR group. Another aspect that we wished to study with this protocol was the timecourse of the effect of euglycemia in restoring the direct effect. Once euglycemia was achieved between 90 and $105 \mathrm{~min}$, the GP suppression curves significantly diverged 
overtime. This time-course is consistent with an acute effect of correction of hyperglycemia on GP.

\section{Protocol 2}

A significantly lower level of insulin in the peripheral circulation was needed with POR-REPL than PER-REPL. This finding suggests that POR-REPL achieved the same glucose levels because of an effect of the greater hepatic insulinization on suppressing GP. This direct effect was found $\sim 60$ min after achievement of euglycemia (as our first basal sample was taken after $1 \mathrm{~h}$ euglycemia). The insulin dose required to achieve euglycemia was $\sim 50 \%$ of the portal dose, which suggests that this direct effect was small. However, since the insulin extraction in these dogs was $\sim 60 \%$, for no direct effect to be present, the PER-REPL dose would have had to be $\sim 40 \%$ of the POR-REPL dose.

GP and GU were the same with both POR-REPL and PER-REPL. This is surprising because, with higher peripheral and expected lower portal insulin levels in PERREPL, a higher $\mathrm{GP}=\mathrm{GU}$ was expected. However, it is likely that greater peripheral insulin levels in PER-REPL inhibited GP by an indirect mechanism but did not modify GU. This is not surprising since we have shown that small increases in peripheral insulin levels above basal do not affect GU in these dogs (Giacca et al. 1999).

Of the possible mediators in the indirect mechanism of GP suppression by insulin, FFAs tended to be lower, but were not significantly affected by PER-REPL, whereas glucagon was not changed. Our finding of indirect effects of insulin on suppressing GP in the absence of changes in FFA and glucagon indicates alternate mechanisms for suppression of $\mathrm{GP}$ by insulin. One potential site of the indirect suppression of GP is the brain (Obici et al. 2002), although the central action of insulin to suppress GP acutely has been questioned in the dog (Edgerton et al. 2006).

The present results show that the peripheral route of insulin treatment can induce peripheral hyperinsulinemia and that euglycemia can be achieved with less systemic insulin levels with portal versus peripheral acute insulin treatment. We have shown in streptozotocin diabetic rats that GP is suppressed to a greater extent following chronic i.p. than s.c. insulin treatment despite marked peripheral hyperinsulinemia with s.c. treatment (Wan et al. 2000). This suggests that the direct effect of insulin is more accentuated during chronic insulin treatment, probably due to insulin's effect on the gene expression and activity of hepatic enzymes (Mason et al. 2000).

\section{Conclusions}

In the present study, we have shown that, in moderately hyperglycemic depancreatized dogs, portal insulin infusion appears to be more effective than peripheral insulin infusion in suppressing GP once euglycemia is achieved. Furthermore, under conditions of euglycemia, less peripheral insulin was required to match glucose and glucose production levels with portal replacement of insulin than with peripheral replacement of insulin, which suggests that portal replacement of insulin achieved the same glucose levels via a direct effect of greater hepatic insulinization on glucose production. These studies clearly show that the direct effect of insulin in suppressing glucose production is present in a model of type 1 diabetes under euglycemic conditions and that its deficiency during peripheral insulin delivery can account in part for the peripheral hyperinsulinemia of insulin-treated diabetes. Based on these findings, future studies aimed at producing safe and relatively non-invasive portal insulin delivery systems are warranted.

\section{Acknowledgements}

The authors thank L Lam for excellent technical assistance. This study was supported by grants to A G from the Juvenile Diabetes Foundation and the Canadian Diabetes Association. Support for personnel was also provided by the Canadian Institutes of Health Research (formerly Medical Research Council of Canada) and the Heart and Stroke Foundation of Canada. The authors declare that there is no conflict of interest that would prejudice the impartiality of this scientific work.

\section{References}

Ader M \& Bergman RN 1990 Peripheral effects of insulin dominate suppression of fasting hepatic glucose production. American Journal of Physiology 258 E1020-E1032.

Bjorkman O, Miles P, Wasserman D, Lickley L \& Vranic M 1988 Regulation of glucose turnover during exercise in pancreatectomized, totally insulindeficient dogs. Effects of beta-adrenergic blockade. Journal of Clinical Investigation 81 1759-1767.

Bradley DC, Steil GM \& Bergman RN 1993 Quantitation of measurement error with optimal segments: basis for adaptive time course smoothing. American Journal of Physiology 264 E902-E911.

Despres JP, Lamarche B, Mauriege P, Cantin B, Dagenais GR, Moorjani S \& Lupien PJ 1996 Hyperinsulinemia as an independent risk factor for ischemic heart disease. New England Journal of Medicine 334 952-957.

Edgerton DS, Lautz M, Scott M, Everett CA, Stettler KM, Neal DW, Chu CA \& Cherrington AD 2006 Insulin's direct effects on the liver dominate the control of hepatic glucose production. Journal of Clinical Investigation 116 521-527.

Finegood DT, Bergman RN \& Vranic M 1987 Estimation of endogenous glucose production during hyperinsulinemic-euglycemic glucose clamps. Comparison of unlabeled and labeled exogenous glucose infusates. Diabetes 36 914-924.

Giacca A, Fisher SJ, Shi ZQ, Gupta R, Lickley HL \& Vranic M 1992 Importance of peripheral insulin levels for insulin-induced suppression of glucose production in depancreatized dogs. Journal of Clinical Investigation 90 1769-1777.

Giacca A, Fisher SJ, McCall RH, Shi ZQ \& Vranic M 1997 Direct and indirect effects of insulin in suppressing glucose production in depancreatized dogs: role of glucagon. Endocrinology 138 999-1007.

Giacca A, McCall R, Chan B \& Shi ZQ 1999 Increased dependence of glucose production on peripheral insulin in diabetic depancreatized dogs. Metabolism 48 153-160. 
Giovannucci E 2001 Insulin, insulin-like growth factors and colon cancer: a review of the evidence. Journal of Nutrition 131 3109S-3120S.

Gupta N, Sandhu H, Goh T, Shah K, Wiesenthal SR, Yoshii H, Chong V, Lam TK, Haber CA, Williams W et al. 2002 Insulin inhibits glucose production by a direct effect in diabetic depancreatized dogs during euglycemia. American Journal of Physiology, Endocrinology and Metabolism 283 E1002-E1007.

Kadish AH \& Sternberg JC 1969 Determination of urine glucose by measurement of rate of oxygen consumption. Diabetes 18 467-470.

Lewis GF, Zinman B, Groenewoud Y, Vranic M \& Giacca A 1996 Hepatic glucose production is regulated both by direct hepatic and extrahepatic effects of insulin in humans. Diabetes 45 454-462.

Lewis GF, Carpentier A, Vranic M \& Giacca A 1999 Resistance to insulin's acute direct hepatic effect in suppressing steady-state glucose production in individuals with type 2 diabetes. Diabetes 48 570-576.

Mason TM, Gupta N, Goh T, El Bahrani B, Zannis J, van de Werve G \& Giacca A 2000 Chronic intraperitoneal insulin delivery, as compared with subcutaneous delivery, improves hepatic glucose metabolism in streptozotocin diabetic rats. Metabolism 49 1411-1416.

McCall RH, Wiesenthal SR, Shi ZQ, Polonsky K \& Giacca A 1998 Insulin acutely suppresses glucose production by both peripheral and hepatic effects in normal dogs. American Journal of Physiology 274 E346-E356.

Miles J, Glasscock R, Aikens J, Gerich J \& Haymond M 1983 A microfluorometric method for the determination of free fatty acids in plasma. Journal of Lipid Research 24 96-99.

Obici S, Zhang BB, Karkanias G \& Rossetti L 2002 Hypothalamic insulin signaling is required for inhibition of glucose production. Nature Medicine 8 1376-1382.

Prager R, Wallace P \& Olefsky JM 1987 Direct and indirect effects of insulin to inhibit hepatic glucose output in obese subjects. Diabetes 36 607-611.

Radziuk J \& Lickley HL 1985 The metabolic clearance of glucose: measurement and meaning. Diabetologia 28 315-322.
Rossetti L, Giaccari A, Barzilai N, Howard K, Sebel G \& Hu M 1993 Mechanism by which hyperglycemia inhibits hepatic glucose production in conscious rats. Implications for the pathophysiology of fasting hyperglycemia in diabetes. Journal of Clinical Investigation 92 1126-1134.

Sindelar DK, Balcom JH, Chu CA, Neal DW \& Cherrington AD 1996 A comparison of the effects of selective increases in peripheral or portal insulin on hepatic glucose production in the conscious dog. Diabetes $\mathbf{4 5}$ 1594-1604.

Sindelar DK, Chu CA, Neal DW \& Cherrington AD 1997 Interaction of equal increments in arterial and portal vein insulin on hepatic glucose production in the dog. American Journal of Physiology 273 E972-E980.

Sindelar DK, Chu CA, Venson P, Donahue EP, Neal DW \& Cherrington AD 1998 Basal hepatic glucose production is regulated by the portal vein insulin concentration. Diabetes 47 523-529.

Staehr P, Hother-Nielsen O, Levin K, Holst JJ \& Beck-Nielsen H 2001 Assessment of hepatic insulin action in obese type 2 diabetic patients. Diabetes 50 1363-1370.

Wan CK, Giacca A, Matsuhisa M, El Bahrani B, Lam L, Rodgers C \& Shi ZQ 2000 Increased responses of glucagon and glucose production to hypoglycemia with intraperitoneal versus subcutaneous insulin treatment. Metabolism 49 984-989.

Received 22 July 2005

Received in final form 25 May 2006

Accepted 6 June 2006

Made available online as an Accepted Preprint 17 July 2006 